\section{Asociación entre adicción a la comida y estado nutricional en universitarios chilenos}

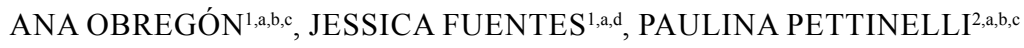

\section{Association between food addiction and nutritional status in Chilean college students}

Background: High calorie foods, especially high in sugar and sodium may have an addictive potential. Experimental rats are able to develop symptoms and neurochemical changes, comparable to those observed in drug abuse, when they are exposed intermittently to sucrose. Aim: To evaluate the association between nutritional status and the prevalence of food addiction in Chilean college students. Material and Methods: Food addiction was assessed using the Yale Food Addiction Scale in 292 Chilean students aged 18-39 years (35\% males). Height and weight were measured and body mass index (weight/height ${ }^{2}$ ) was calculated. Results: Eleven percent of participants met the criteria for food addiction. Women had a higher prevalence than men (14.4\% and $4.8 \%$, respectively). Thirty percent of individuals with a body mass index over $30 \mathrm{~kg} / \mathrm{m}^{2}$ met the criteria for food addiction. Conclusions: In these Chilean students, food addiction was more prevalent in women and a higher prevalence was observed in obese individuals.

(Rev Med Chile 2015; 143: 589-597)

Key words: Addiction; Feeding Behavior; Obesity.
${ }^{1}$ Carrera de Nutrición y Dietética, Facultad de Ciencias de la Salud. Universidad San Sebastián, Concepción, Chile.

${ }^{2}$ Ciencias de la Salud, Nutrición y Dietética, Facultad de Medicina. Pontificia Universidad Católica de Chile, Santiago, Chile.

${ }^{a}$ Nutricionista.

bPhD Nutrición y Alimentación.

'Magíster en Ciencias Biológicas Mención Nutrición.

${ }^{\mathrm{d}}$ Magíster en Salud Pública.

Fuente de financiamiento: Universidad San Sebastián. Conflictos de intereses: ninguno que declarar.

Recibido el 22 de abril de 2014, aceptado el 13 de enero de 2015

Correspondencia a:

Prof. Ana M. Obregón PhD, MSc. Escuela de Nutrición y Dietética, Facultad de Ciencias de la Salud, Universidad San Sebastián. General Cruz 1577, Concepción, Chile.

Teléfono: 56-41-2487254 ana.obregon@uss.cl

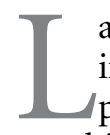

a obesidad resulta de un desbalance entre la ingesta y el gasto energético que se produce por la interacción entre factores biológicos $\mathrm{y}$ ambientales que favorecen un exceso de ingesta $^{1,2}$. Dentro de los factores condicionantes del peso corporal se encuentran la disminución de la actividad física y el sobreconsumo de alimentos de alta palatabilidad y elevada densidad energética. De éstos, el sobreconsumo de alimentos se describe como el preponderante. Este puede ocurrir en algún grado en todos los individuos; no obstante, una proporción de ellos puede desarrollar un consumo obsesivo compulsivo hacia ciertos alimentos ${ }^{3}$. Estos individuos consumen crónicamente mayores cantidades de alimentos que los que requieren para mantener la salud y muestran comportamientos de ingesta compulsiva y pérdida de control sobre los alimentos ${ }^{4,5}$.

En 1956, Randolph describió la existencia de individuos que presentaban síntomas relacionados con los alimentos, con un patrón similar a lo observado en la adicción. Dentro de esta sintomatología se observaba una adaptación a ciertos alimentos consumidos regularmente. Posteriormente, modelos adictivos fueron descritos para el consumo de maíz, avena, leche, huevos y papas $^{6}$. Desde entonces, se desarrolló una teoría que plantea que alimentos altos en calorías, ricos en azúcares, podrían tener un potencial adicti$\mathrm{vo}^{7,8}$. La repetida exposición a ciertos alimentos, particularmente alimentos de elevada densidad energética, en individuos vulnerables puede desencadenar un consumo compulsivo y un bajo control sobre la ingesta.

Se han estudiado paralelismos entre la dependencia de sustancias y el excesivo consumo de alimentos hiper-palatables ${ }^{10}$. Debido a esto, se ha argumentado que la obesidad debiera ser 
considerada como un desorden a nivel cerebral, e incluida como categoría diagnóstica en el Manual Diagnóstico y Estadístico de Trastornos Mentales $(\mathrm{DSM}-\mathrm{V})^{9,10}$.

Estudios en animales muestran que cuando las ratas tienen acceso a azúcar, grasa o alimentos procesados, desarrollan una alteración en mecanismos neuronales relacionados con el refuerzo que están involucrados en la adicción ${ }^{7,11}$. También se ha observado que desarrollan comportamientos adictivos como tolerancia, abstinencia, consumo excesivo y uso continuo de una sustancia, a pesar de presentar problemas físicos (shocks eléctricos $)^{7,11}$. En humanos, la obesidad y la dependencia de sustancias ha sido relacionada con algunos marcadores neurológicos, como menores niveles de expresión cerebral del receptor 2 de dopamina DRD2 ${ }^{12}$. El fundamento biológico de la adición a la comida proviene de que tanto el consumo de alimentos como el consumo de drogas desencadenan la liberación de dopamina en regiones meso límbicas (corteza orbito frontal [COF], amígdala, ínsula, stratium y corteza cingulada anterior [CCA]) y el grado de liberación tiene una correlación positiva con el grado de refuerzo subjetivo de los alimentos y drogas ${ }^{12}$. Estudios en humanos muestran que individuos con dependencia de sustancias tienen mayor activación en regiones cerebrales que codifican el valor del refuerzo de un determinado estímulo (ej.: COF, amígdala, ínsula, stratium y CCA) en comparación a individuos sin dependencia. Concordante con esto, se ha observado que individuos obesos versus normopeso muestran mayor activación en la COF, amígdala, CCA, stratium, e hipotálamo medio-dorsal, en respuesta a estímulos de alimentos $^{12}$. Para poder evaluar este rasgo, Gearhardt y cols (2009) desarrollaron un instrumento psicométrico (Yale Food Addiction Scale; YFAS) basado en el DSM-IV-TR para operacionalizar el constructo de "adicción a la comida". Este cuestionario permite diferenciar individuos con y sin un patrón adictivo a una determinada sustancia (alimentos de alta palatabilidad) ${ }^{13}$.

El uso de este instrumento muestra que mayores puntajes de adicción a la comida se correlacionan con una mayor activación de regiones que están implicadas en la dependencia de sustancias, en respuesta a determinados alimentos ${ }^{14,15}$. Utilizando este cuestionario se ha explorado la prevalencia de "adicción a la comida" en pacientes con desórdenes alimentarios ${ }^{16}$, pacientes obesos ${ }^{17} \mathrm{y}$ universitarios $^{18}$. El objetivo de esta investigación fue evaluar la asociación entre el estado nutricional y la prevalencia de la adicción a la comida en universitarios chilenos.

\section{Individuos y Método}

Estudio de corte transversal en 292 universitarios chilenos de 18 a 39 años de edad. Los individuos fueron invitados a participar mediante ficheros o invitación personalizada y firmaron un consentimiento informado. El estudio se llevó a cabo siguiendo las normas de la Declaración de Helsinki y tras haber obtenido la aprobación del Comité de Ética de la Universidad San Sebastián.

\section{Antropometría}

Se midió el peso y la talla utilizando una balanza SECA (Modelo 700) mediante procedimientos estándar ${ }^{19}$. La clasificación del estado nutricional se realizó mediante el cálculo del índice de masa corporal (IMC: $\mathrm{Kg} / \mathrm{m}^{2}$ ) utilizando el criterio propuesto por la OMS $(2004)^{20}$.

\section{Adicción a la comida}

Fue evaluada utilizando el cuestionario de adicción a la comida (YALE-FAS: Yale Food Addiction Scale $)^{14}$. Este instrumento fue solicitado a los autores, quienes autorizaron su uso en población chilena. Se realizó una adaptación local del cuestionario original mediante una traducción directa-reversa. A continuación, se realizó un estudio piloto en 40 universitarios de ambos sexos para analizar la comprensión del instrumento. El YALE-FAS fue aplicado por nutricionistas y alumnos entrenados de la carrera de Nutrición y Dietética de la Universidad San Sebastián. El estudio se llevó a cabo en una sala cómoda adaptada para los objetivos. El cuestionario YALE-FAS consta de 25 preguntas relacionadas con la conducta alimentaria hacia alimentos de alta palatabilidad (grasas/ altos en carbohidratos) en los últimos 12 meses e incluye categorías de respuestas mixtas (Respuestas dicotómicas/Escala de Likert). El YALE-FAS es un instrumento desarrollado para identificar individuos propensos a presentar síntomas de dependencia de consumo de alimentos basado en los criterios de dependencia de sustancias del DSM-IV (Tabla 1). Adicionalmente, incorpora 2 preguntas que evalúan la presencia de alteración 
Tabla 1. Criterio diagnóstico para dependencia de sustancias de acuerdo a DSM-IV

1. Tomar sustancia en grandes cantidades o durante un período más prolongado de los que se requiere

2. Deseo persistente o esfuerzos infructuosos por controlar o interrumpir el consumo de la sustancia

3. Empleo de mucho tiempo en actividades relacionadas con la obtención y consumo de la sustancia o recuperación de sus efectos

4. Reducción o abandono de importantes actividades sociales, laborales o recreativas debido al consumo de sustancias

5. Uso continuo de la sustancia a pesar de problemas psicológicos o físicos

6. Tolerancia

7. Síntomas de abstinencia

clínica o distress. El criterio diagnóstico se cumple cuando 3 síntomas se encuentran presentes y adicionalmente se observa alteración clínica o distress.

\section{Estadística}

Las variables paramétricas se presentaron como promedio \pm desviación estándar. Se utili- zó el test $\mathrm{t}$ de Student para la comparación por género. Se consideraron significativos los valores de $\mathrm{p} \leq 0,05$. El análisis estadístico se realizó con el programa STATA 13.0. Utilizando los valores de IMC $\left(\mathrm{kg} / \mathrm{m}^{2}\right)$ como variable predictora y la presencia de adicción a la comida como variable respuesta se realizó una regresión logística para buscar asociación entre el estado nutricional y la adicción a la comida.

\section{Análisis factorial y consistencia interna}

El análisis factorial exploratorio se apoyó en la prueba de adecuación muestral de Kaiser Meyer-Olkin (KMO), que fue de 0,59 y en la prueba de esfericidad de Bartlett, que resultó estadísticamente significativa $\chi^{2}(300)=1.686,3(p<0,05)$. Usando el método de extracción de componentes principales se identificaron 7 componentes con valores propios "eigenvalue" superiores a $1(6,08$, $2,27,1,53,1,35,1,24,1,1,1,01)$ que explicaron $58 \%$ de la varianza total. Aun cuando estos factores mostraron valores propios $\geq 1,0$, el Screen plot sugiere una estructura de 1 factor (Tabla 2, Figura 1). Adicionalmente se realizó un análisis de consistencia interna del instrumento, que mostró un Alfa de Cronbach de 0,87. El análisis de consistencia interna fue consistente con el instrumento original donde se observó un Alfa de Cronbach de $0,86^{14}$.

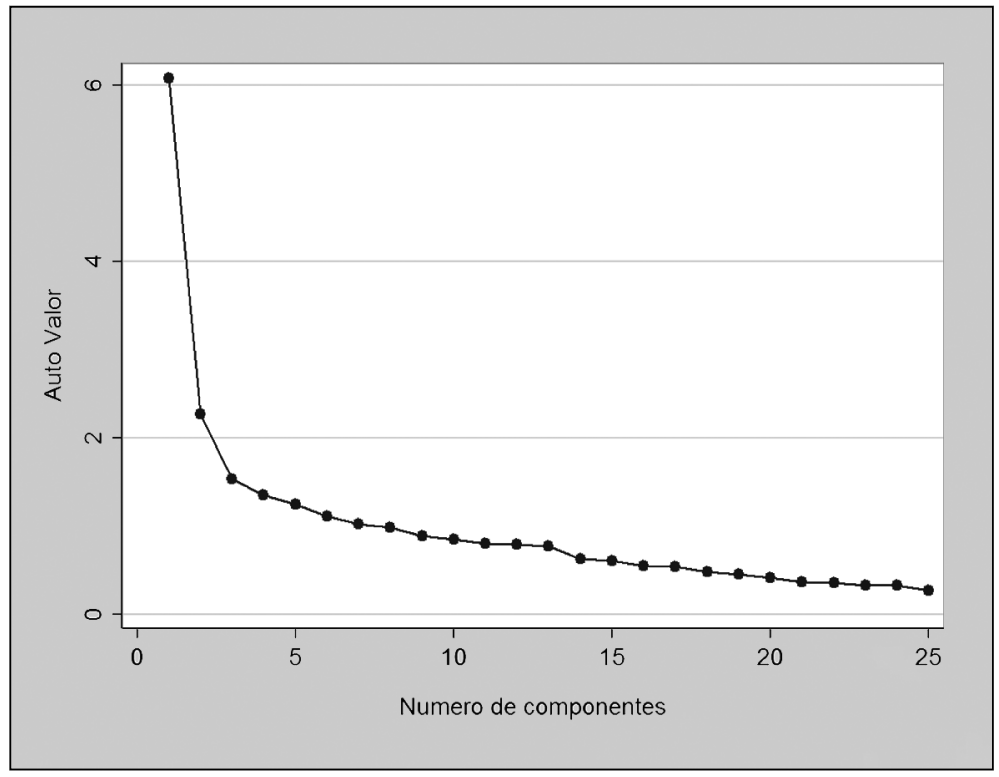

Figura 1. Screen plot de análisis de componentes principales de la escala de adicción a la comida. 


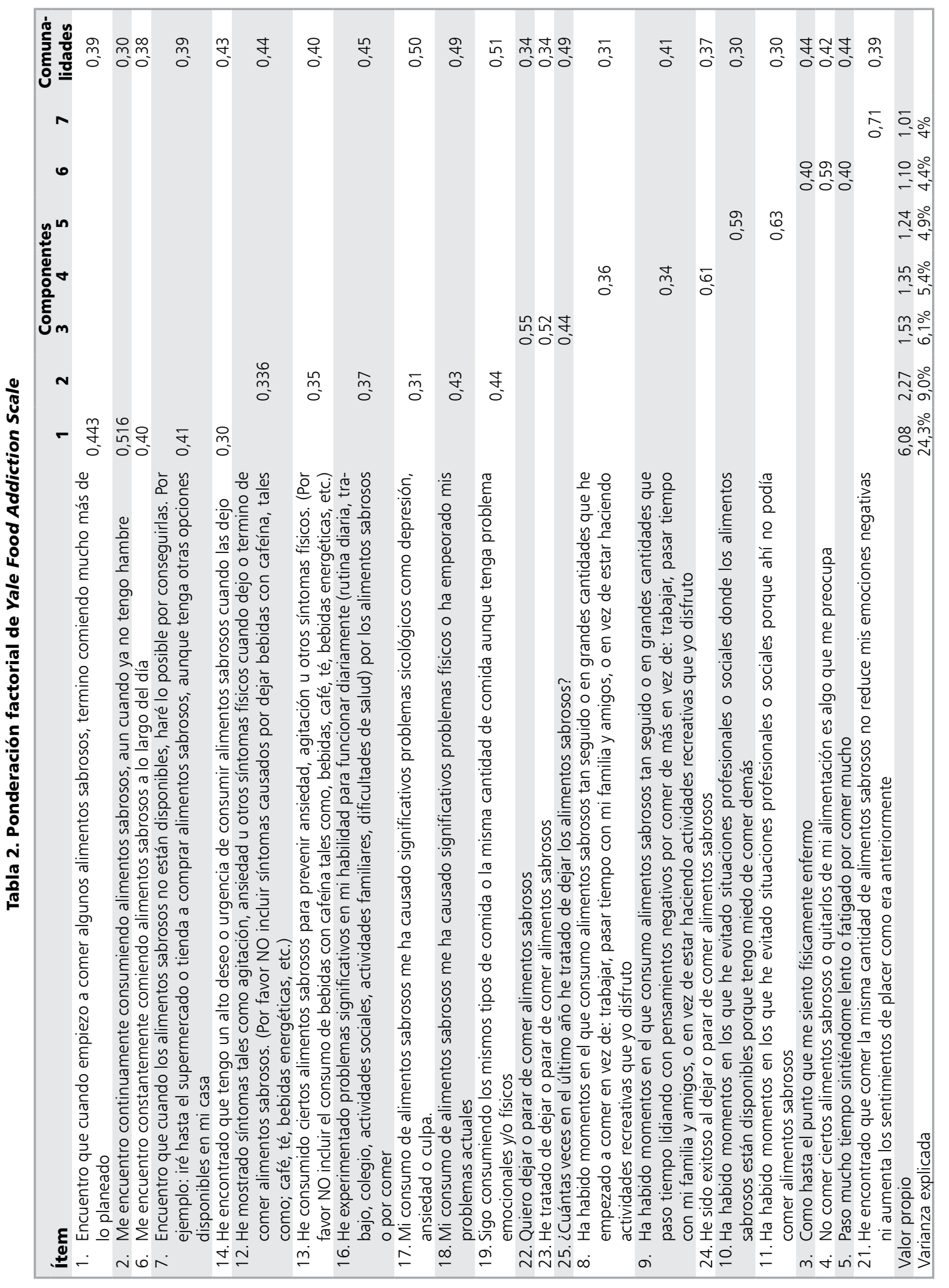


Adicción a la comida y estado nutricional en universitarios - A. Obregón et al

\section{Resultados}

Se reclutaron 292 estudiantes universitarios provenientes de la Región del Bío Bío, Chile (35\% hombres y $64 \%$ mujeres), con un promedio de edad de 21,4 $\pm 2,4$ años. La Tabla 3 muestra las variables antropométricas de los sujetos. Se observó una diferencia significativa en peso y talla por género ( $\mathrm{p}$-value $<0,05$ ). En la muestra se encontró que $2,5 \%$ de los individuos presentaron bajo peso;

Tabla 3. Mediciones antropométricas por género

\begin{tabular}{|lcc|}
\hline & $\begin{array}{c}\text { Hombres } \\
(\mathbf{n}=\mathbf{1 0 5})\end{array}$ & $\begin{array}{c}\text { Mujeres } \\
(\mathbf{n}=\mathbf{1 8 7})\end{array}$ \\
\hline Edad (años) & $21,1 \pm 2,2$ & $21,5 \pm 2,4$ \\
\hline Peso $(\mathrm{Kg})$ & $72,5 \pm 10,7$ & $58,8 \pm 8,4^{*}$ \\
\hline Talla $(\mathrm{cm})$ & $1,75 \pm 0,11$ & $1,6 \pm 0,87^{*}$ \\
\hline IMC $\left(\mathrm{kg} / \mathrm{m}^{2}\right)$ & $23,7 \pm 2,8$ & $22,8 \pm 3,03$ \\
\hline
\end{tabular}

Promedio \pm Desviación Estándar, IMC- Índice de masa corporal. *Diferencia significativa entre hombres y mujeres (t-test $<0,05)$.
$76 \%$ normo peso; $18 \%$ sobrepeso y $3,5 \%$ obesidad. En relación a la adicción a la comida, la mayor parte de los encuestados tuvo de 0-4 síntomas (Tabla 4). Los síntomas presentados con mayor frecuencia fueron: a) Deseo persistente o esfuerzos infructuosos por controlar o interrumpir el consumo de la sustancia (57\%); b) Uso continuo de la sustancia a pesar de problemas psicológicos o físicos $(41,1 \%)$; c) Tolerancia $(36,3 \%)$ y d) Síntomas de abstinencia $(23,9 \%)$ (Tabla 4$)$. En esta muestra exploratoria se encontró que $10,9 \%$ de los individuos cumplieron el criterio diagnóstico. Las mujeres tuvieron mayores prevalencias que los hombres (14,4\% vs $4,78 \%)$. Al analizar por estado nutricional, se observó mayor prevalencia en el grupo de los obesos (30\%), sobrepeso $(13,2 \%)$ y bajo peso $(14,2 \%)$ (Tabla 5). Cuando se analizó la prevalencia por género se observó que en el grupo de las mujeres la prevalencia fue mayor en el grupo de obesas (40\%), luego sobrepeso $(21,4 \%)$, normales $(12,1 \%)$ y finalmente bajo peso $(6,2 \%)$ (Tabla 5). El resultado de la regresión logística mostró una asociación entre el índice

Tabla 4. Prevalencia de síntomas de adicción a la comida

\begin{tabular}{|lcc|}
\hline Criterios de dependencia de sustancia & n & \% \\
$\begin{array}{l}\text { 1. Tomar sustancia en grandes cantidades o durante un período más prolongado de los } \\
\text { que se requiere }\end{array}$ & $59 / 292$ & 20,1 \\
\hline $\begin{array}{l}\text { 2. Deseo persistente o esfuerzos infructuosos por controlar o interrumpir el consumo de } \\
\text { la sustancia }\end{array}$ & $168 / 292$ & 57,5 \\
\hline $\begin{array}{l}\text { 3. Empleo de mucho tiempo en actividades relacionadas con la obtención y consumo de } \\
\text { la sustancia o recuperación de sus efectos }\end{array}$ & $66 / 292$ & 22,6 \\
$\begin{array}{l}\text { 4. Reducción o abandono de importantes actividades sociales, laborales o recreativas } \\
\text { debido al consumo de sustancias }\end{array}$ & $57 / 292$ & 19,5 \\
\hline $\begin{array}{l}\text { 5. Uso continuo de la sustancia a pesar de problemas psicológicos o físicos } \\
\text { 6. Tolerancia }\end{array}$ & $120 / 292$ & 41,1 \\
\hline 7. Síntomas de abstinencia & $106 / 292$ & 36,3 \\
\hline
\end{tabular}

Tabla 5. Porcentaje de adicción a la comida por estado nutricional

\begin{tabular}{|lccccccc|}
\hline & IMC (kg/m $\mathbf{2})$ & $\begin{array}{c}\text { Diagnóstico } \\
\text { Adicción (\%) } \\
\text { Hombres }\end{array}$ & $\begin{array}{c}\text { Tamaño } \\
\text { Muestra }\end{array}$ & $\begin{array}{c}\text { Diagnóstico } \\
\text { Adicción (\%) } \\
\text { Mujeres }\end{array}$ & $\begin{array}{c}\text { Tamaño } \\
\text { Muestra }\end{array}$ & $\begin{array}{c}\text { Diagnóstico } \\
\text { Adicción } \\
\text { Tota) }\end{array}$ & $\begin{array}{c}\text { Tamaño } \\
\text { Muestra } \\
(\mathbf{n}=\mathbf{2 9 2})\end{array}$ \\
\hline Bajo peso & $<18,5$ & 0 & $0 / 1$ & 16,7 & $1 / 6$ & 14,2 & $1 / 7$ \\
\hline Normal & $18,5-24,99$ & 4,0 & $3 / 74$ & 12,1 & $18 / 148$ & 9,46 & $21 / 222$ \\
\hline Sobrepeso & $25,0-29,9$ & 4,0 & $1 / 25$ & 21,4 & $6 / 28$ & 13,2 & $7 / 53$ \\
\hline Obeso & $\geq 30,0$ & 20 & $1 / 5$ & 40 & $2 / 5$ & 30 & $3 / 10$ \\
\hline
\end{tabular}

La clasificación por peso se realizó descuerdo a los patrones de la OMS 2004. 
Tabla 6. Variables asociadas con adicción a la comida

\begin{tabular}{|lccc|}
\hline Variable & Razón de disparidad (OR) & $\mathbf{9 5 \%}$ IC & p \\
\hline Índice de masa corporal $\left(\mathrm{kg} / \mathrm{m}^{2}\right)$ & 1,73 & $1,29-2,33$ & 0,001 \\
\hline Edad (años) & 0,966 & $-0,39-0,696$ & 0,69 \\
\hline Sexo & 0,412 & $0,97-1,75$ & 0,23 \\
\hline
\end{tabular}

de masa corporal y la presencia o no de adicción a la comida. Se obtuvo que por cada unidad de aumento en el índice de masa corporal el odds de padecer adicción a la comida aumentó en 1,73 (OR $=1,7 ; 95 \%$ IC: $1,29-2,33 \mathrm{p}<0,05)$ (Tabla 6). Adicionalmente se observó que la adicción a la comida se correlaciona positivamente con el IMC $(\mathrm{r}=0,17 ; \mathrm{p}=0,002)$.

\section{Discusión}

La obesidad resulta de una interacción entre múltiples loci genéticos y el ambiente en el cual los individuos están expuestos ${ }^{21}$. Existe un consenso que plantea que un ambiente permisivo de alimentos es un importante factor de riesgo que contribuye al desarrollo de esta enfermedad ${ }^{22}$. Sin embargo, cabe destacar que no todos los individuos desarrollan esta condición, existiendo una gran variabilidad individual en el peso corporal y en la ganancia de peso en el tiempo ${ }^{23}$. El factor más preponderante de la obesidad es el excesivo consumo de energía y macronutrientes. En relación a esto, se ha establecido que la conducta alimentaria de los individuos influye en gran medida la selección y la cantidad de alimentos ingeridos ${ }^{24}$. Las diferencias individuales de la conducta alimentaria han sido evaluadas midiendo una serie de sub-conductas de la alimentación dentro de las cuales se encuentra la adicción a la comida ${ }^{25}$.

En este estudio exploratorio se evaluó por primera vez en Chile la prevalencia de adicción a la comida en universitarios chilenos utilizando el instrumento YFAS. Los resultados de esta investigación mostraron una prevalencia de adicción a la comida cercana a $10 \%$, siendo mayor para el género femenino. Estos datos son concordantes con lo observado por Gearhardt et al. en estudiantes universitarios, donde se observó que $11,4 \%$ cumple el criterio diagnóstico ${ }^{14}$. Otros estudios han mostrado resultados similares. En este sentido, Meule et al. reportaron una prevalencia de $8,8 \%{ }^{26} \mathrm{y}$, además, encontraron una leve correlación positiva entre los síntomas del YFAS y el IMC. Adicionalmente, otros autores han descrito prevalencias mayores, cercanas a $25 \%{ }^{18}$. El análisis de factores realizado para el instrumento aplicado a una muestra chilena, identificó una estructura de un solo factor y una adecuada consistencia interna, en concordacia con el instrumento original ${ }^{13}$. Este rasgo también ha sido explorado en niños con sobrepeso y obesidad, donde se ha mostrado que $29 \%$ refieren ser adictos a ciertos tipos de alimentos ${ }^{27}$.

Adicionalmente, Merlo et al. ${ }^{28}$ encontraron que $15,2 \%$ de los niños con sobrepeso indican que "siempre" y "casi siempre" sienten ser adictos a los alimentos. Otro aspecto relevante es que la prevalencia de adicción a la comida difiere según el estado nutricional. En relación a esto, Meule et al., en una muestra de adultos, encontraron diferente prevalencia en individuos obesos $(37,5 \%)$, sobrepeso $(14 \%)$ y normales $(6,3 \%)^{29}$.

En nuestro estudio se encontró una mayor prevalencia en obesos que en individuos con peso normal; sin embargo, una limitante de nuestra muestra fue el que haya existido un bajo número de obesos (3/10) dado que se trataba de universitarios. Adicionalmente la muestra fue en sujetos voluntarios lo que hace que los resultados no puedan ser generalizados hacia toda la población de universitarios. Sería interesante en el futuro tomar un número mayor de adultos donde se incluyan individuos de todas las edades.

Los principales síntomas descritos en la literatura al evaluar la adicción a la comida son: 1) deseo persistente o esfuerzos infructuosos por controlar o interrumpir el consumo de la sustancia; 2) uso continuo de la sustancia a pesar de problemas psicológicos o físicos; y 3) empleo de mucho tiempo en actividades relacionadas con la obtención y consumo de la sustancia o recuperación de sus efectos ${ }^{29}$. Recientemente se ha mostrado que 
estos 3 síntomas fueron presentados en pacientes obesos con Binge eating disorder (BED) ${ }^{29}$ Estos resultados son concordantes con lo observado en nuestra investigación.

La comprensión del sobreconsumo como un potencial desorden adictivo proviene de estudios en animales y estudios de neuro-imágenes realizados en humanos ${ }^{30,31}$. Estos estudios muestran que los alimentos de alta palatabilidad, altos en azúcar, grasa y sal, tienen efectos similares al de algunas drogas adictivas, en el cerebro ${ }^{30}$. En este sentido, individuos adictos a drogas, consumidores compulsivos e individuos obesos muestran reducciones en la actividad dopaminérgica, pobre control inhibitorio y reducida sensibilidad al placer ${ }^{30,32}$. Un estudio realizado por Wang et al. mostró que los sujetos obesos mórbidos (IMC $>40 \mathrm{~kg} / \mathrm{m}^{2}$ ), tenían menor disponibilidad del receptor 2 de dopamina DRD2, proporcional al grado de obesidad ${ }^{33}$. En otras palabras, los sujetos con menor densidad de receptores tenían mayor $\mathrm{IMC}^{33,34}$. Esto es consistente con los hallazgos de que el bloqueo de DRD2 (medicamentos antipsicóticos) incrementa el consumo energético ${ }^{35}$.

Se han encontrado similitudes y paralelos en las vías de funcionamiento neuronal, entre la obesidad y la dependencia de sustancias (drogas) ${ }^{12}$. Se ha descrito que el sobreconsumo de alimentos y drogas son regidos por las propiedades reforzadoras o gratificantes, las que envuelven el sistema dopaminérgico ${ }^{36}$. De esta manera, los alimentos activan el refuerzo cerebral por medio de la palatabilidad (mecanismo que envuelve opioides y canabinoides endógenos) y por medio del aumento de la glucosa e insulina (produciendo aumento de dopamina), mientras que las drogas activan el mismo circuito por una vía farmacológica (efectos directos en células dopaminérgicas). Se cree que la repetida estimulación de la vía del refuerzo mediada por dopamina desencadena adaptaciones neurobiológicas en otros neurotransmisores y circuitos que conllevan a aumentar el comportamiento compulsivo y pérdida de control sobre alimentos y drogas. Se ha estudiado que reducciones en los niveles del receptor 2 de dopamina stratial, se asocian con disminución de actividad metabólica en la COF, corteza gyrus (CG) y corteza dorso lateral $(\mathrm{CDL})^{37}$. Dado que estas regiones están involucradas en el control inhibitorio ${ }^{38}$ y en el procesamiento emocional ${ }^{39}$, se ha postulado que una desregulación por dopamina, puede llevar a pérdida de control sobre la ingesta y alteración en la emociones ${ }^{40}$.

En conclusión, hemos comunicado el primer estudio exploratorio de prevalencia de adicción a la comida en población chilena, medido a través de YFAS. Se observó que esta condición es más prevalente en mujeres que en hombres y que está relacionada con la obesidad. Se requiere más investigación para poder comprender las potenciales causas de esta condición. Estos resultados pueden contribuir a entender los factores que influyen en el desarrollo de la obesidad.

Agradecimientos: A los alumnos de la Carrera de Nutrición y Dietética que colaboraron en la recopilación de los datos.

\section{Referencias}

1. Pataky Z, Bobbioni-Harsch E, Golay A. Obesity: a complex growing challenge. Exp Clin Endocrinol Diabetes 2010; 118 (7): 427-33. doi: 10.1055/s-0029-1233448.

2. Swinburn BA, Sacks G, Hall KD, McPherson K, Finegood DT, Moodie ML, et al. The global obesity pandemic: shaped by global drivers and local environments. Lancet 2011 27; 378 (9793): 804-14. doi: 10.1016/S01406736(11)60813-1.

3. Ifland JR, Preuss HG, Marcus MT, Rourke KM, Taylor WC, Burau K, et al. Refined food addiction: a classic substance use disorder. Med Hypotheses 2009; 72 (5): 518-26. doi: 10.1016/j.mehy.2008.11.035.

4. Ziauddeen H, Farooqi IS, Fletcher PC. Obesity and the brain: how convincing is the addiction model? Nat Rev Neurosci 2012 14; 13 (4): 279-86. doi: 10.1038/nrn3212.

5. Barry D, Clarke M, Petry NM. Obesity and its relationship to addictions: is overeating a form of addictive behavior? Am J Addict 2009; 18 (6): 439-51. doi: 10.3109/10550490903205579. Review.

6. Randolph TG. The descriptive features of food addiction-addictive eating and drinking. Q J Stud Alcohol 1956; 17: 198-224.

7. Avena NM, Rada P, Hoebel BG. Evidence for sugar addiction: behavioral and neurochemical effects of intermittent, excessive sugar intake. Neurosci Biobehav Rev 2008; 32 (1): 20-39.

8. Avena NM, Bocarsly ME, Hoebel BG. Animal models of sugar and fat bingeing: relationship to food addiction and increased body weight. Methods Mol Biol. 2012; 829: 351-65. doi: 10.1007/978-1-61779-458-2_23.

9. Volkow ND, O'Brien CP. Issues for DSM-V: should 
obesity be included as a brain disorder? Am J Psychiatry 2007; 164 (5): 708-10.

10. Devlin MJ. Is there a place for obesity in DSM-V? Int J Eat Disord 2007; 40: 83-8.

11. Johnson PM, Kenny PJ. Dopamine D2 receptors in addiction-like reward dysfunction and compulsive eating in obese rats. Nat Neurosci 2010; 13 (5): 635-41. doi: 10.1038/nn.2519.

12. Volkow ND, Wang GJ, Fowler JS, Telang F. Overlapping neuronal circuits in addiction and obesity: evidence of systems pathology. Philos Trans R Soc Lond B Biol Sci. 2008; 363 (1507): 3191-200.

13. Gearhardt AN, Corbin WR, Brownell KD. Preliminary validation of the Yale Food Addiction Scale. Appetite 2009; 52 (2): 430-6. doi: 10.1016/j.appet.2008.12.003.

14. Gearhardt AN, Yokum S, Orr PT, Stice E, Corbin WR, Brownell KD. Neural correlates of food addiction. Arch Gen Psychiatry 2011; 68 (8): 808-16. doi: 10.1001/archgenpsychiatry.2011.32.

15. Clark SM, Saules KK. Validation of the Yale Food Addiction Scale among a weight-loss surgery population. Eat Behav 2013; 14 (2): 216-9. doi: 10.1016/j. eatbeh.2013.01.002.

16. Gearhardt AN, White MA, Masheb RM, Morgan PT, Crosby RD, Grilo CM. An examination of the food addiction construct in obese patients with binge eating disorder. Int J Eat Disord 2012; 45 (5): 657-63. doi: 10.1002/eat.20957.

17. Davis C, Curtis C, Levitan RD, Carter JC, Kaplan AS, Kennedy JL. Evidence that 'food addiction' is a valid phenotype of obesity. Appetite 2011; 57 (3): 711-7. doi: 10.1016/j.appet.2011.08.017.

18. Meule A, Kübler A. Food cravings in food addiction: the distinct role of positive reinforcement. Eat Behav 2012; 13 (3): 252-5. doi: 10.1016/j.eatbeh.2012.02.001.

19. Gordon C, Chumlea W, Roche A. 1991. Stature, recumbent length and weight. In: Lohman T, Roche A, Martorell R. Anthropometric standarization reference manual. Abridged Edition, 3-8.

20. Organización Mundial de la Salud: Appropriate bodymass index for Asian populations and its implications for policy and intervention strategies. Ginebra (Suiza): Organización Mundial de la Salud, 2004.

21. Adamo KB1, Tesson F. Genotype-specific weight loss treatment advice: how close are we? Appl Physiol Nutr Metab 2007; 32 (3): 351-66.

22. French SA, Story M, Jeffery RW. Environmental influences on eating and physical activity. Annu Rev Public Health 2001; 22: 309-35.

23. French SA, Jeffery RW, Folsom AR, Williamson DF, Byers T. Weight variability in a population based sam- ple of older women: Intercorrelation and reliability of measures. Int J Obesity 1995; 19: 22-9.

24. Domínguez-Vásquez P, Olivares S, Santos JL. Eating behavior and childhood obesity: family influences. Arch Latinoam Nutr 2008; 58 (3): 249-55.

25. French SA, Epstein LH, Jeffery RW, Blundell JE, Wardle J. Eating behavior dimensions. Associations with energy intake and body weight. A review. Appetite 2012; 59 (2): 541-9. doi: 10.1016/j.appet.2012.07.001.

26. Meule A, Vögele C, Kübler A. German translation and validation of the Yale Food Addiction Scale. Diagnostica 2012; 58: 115-26.

27. Pretlow R. Addiction to highly pleasurable food as a cause of the childhood obesity epidemic: a qualitative Internet study. Eat Disord 2011; 19 (4): 295-307. doi:10 $.1080 / 10640266.2011 .584803$.

28. Merlo LJ, Klingman C, Malasanos TH, Silverstein J. Exploration of food addiction in pediatric patients: A preliminary investigation. J Addict Med 2009; 3 (1): 2632. doi: 10.1097/ADM.0b013e31819638b0.

29. Meule A. How Prevalent is "Food Addiction"? Front Psychiatry 2011; 2: 61. doi: 10.3389/fpsyt.2011.00061.

30. Lee NM, Carter A, Owen N, Hall WD. The neurobiology of overeating. Treating overweight individuals should make use of neuroscience research, but not at the expense of population approaches to diet and lifestyle. EMBO Rep 2012; 13 (9): 785-90. doi: 10.1038/embor.2012.115.

31. Avena NM, Gold JA, Kroll C, Gold MS. Further developments in the neurobiology of food and addiction: Update on the state of the science. Nutrition 2012; 28: 341-3.

32. Volkow ND, Wang GJ, Baler RD. Reward, dopamine and the control of food intake: implications for obesity. Trends Cogn Sci 2011; 15 (1): 37-46. doi: 10.1016/j. tics.2010.11.001.

33. Wang GJ, Volkow ND, Logan J, Pappas NR, Wong CT, Zhu W, et al. Brain dopamine and obesity. Lancet 2001 3; 357 (9253): 354-7.

34. Haltia LT, Rinne JO, Merisaari H, Maguire RP, Savontaus E, Helin S, et al. Effects of intravenous glucose on dopaminergic function in the human brain in vivo. Synapse 2007; 61 (9): 748-56.

35. Allison DB, Mentore JL, Heo M, Chandler LP, Cappelleri JC, Infante MC, et al. Antipsychotic-induced weight gain: a comprehensive research synthesis. Am J Psychiatry 1999; 156 (11): 1686-96. Review

36. Volkow ND, Wise RA. How can drug addiction help us understand obesity? Nat Neurosci 2005; 8 (5): 55560.

37. Volkow ND, Fowler JS, Wang GJ, Hitzemann R, Logan J, Schlyer DJ, et al. Decreased dopamine D2 receptor 
availability is associated with reduced frontal metabolism in cocaine abusers. Synapse 1993; 14 (2): 169-77. doi:10.1002/syn.890140210.

38. Goldstein RZ, Volkow ND. Drug addiction and its underlying neurobiological basis: neuroimaging evidence for the involvement of the frontal cortex. Am J Psychiatry 2002; 159 (10): 1642-52. doi:10.1176/appi. ajp.159.10.1642.

39. Phan KL, Wager T, Taylor SF, Liberzon I. Functional neuroanatomy of emotion: a meta-analysis of emotion activation studies in PET and fMRI. Neuroimage 2002; 16 (2): 331-48.http://dx.doi.org/10.1006/ nimg.2002.1087.

40. Gearhardt AN, White MA, Masheb RM, Grilo CM. An examination of food addiction in a racially diverse sample of obese patients with binge eating disorder in primary care settings. Compr Psychiatry 2013; 54 (5): 500-5. doi: 10.1016/j.comppsych.2012.12.009. 